\title{
OCT angiography as a novel diagnostic possibility in age-related macular degeneration
}

\author{
Katarzyna Michalska-Małecka, ${ }^{1,2}$, Justyna Sierocka-Stępieńn', Katarzyna Witek², \\ Aneta Michalik-Jakubek ${ }^{2}$ \\ 'Department of Ophthalmology, School of Medicine in Katowice, Medical University of Silesia, Katowice, Poland \\ ${ }^{2}$ University Clinical Centre, University Hospital Medical University of Silesia, Katowice, Poland
}

\begin{abstract}
INTRODUCTION. The aim of the study is to compare classical vein imaging methods used to diagnose Age-related Macular Degeneration (AMD), like fluorescein angiography (FA), with non-invasive modern methods of ultrafast optical blood flow detection (Optical Coherence Tomography-angiography, Angio-OCT).

METHODS. Images obtained during examination using AF and Angio-OCT methods of four randomly selected patients treated for AMD in the Department of Ophthalmology Medical University of Silesia, Katowice, Poland were used for evaluation.

RESULTS. Images obtained during routine check-up of patients treated for AMD were compared.

CONCLUSIONS. Angio-OCT is a non-invasive, non-contact, fast, and safe examination of patients treated for AMD. Angio-OCT is a modern method that provides important information about the progression of the disease, and it is used to evaluate retinal and choroidal vessels in patients with AMD.
\end{abstract}

KEY WORDS: age-related macular degeneration, angiography, optical coherence tomography, imaging, fluorescein

Ophthalmol J 2016; Vol. 1, No. 3, 83-88

\section{INTRODUCTION}

Age-related macular degeneration (AMD) is the leading cause of vision loss that affects people over 50 years of age, occurring in $8.8 \%$ of the population, more frequently among women $[1,2]$. It is estimated that every tenth person over 45 years old is in the risk group of this disease. Optical Coherence Tomography (OCT) is an imaging method that relies on receiving and processing of optical signals originating from sources that emit light having a wide range of frequencies. OCT is a safe and non-invasive diagnostic method that does not require any substance to be added to the human body and does not expose it to radiation. Currently, OCT is used among other diagnostic tools for obtaining high-quality images of anterior segment of the eye and retinal disorders associated with the formation of fluid spaces (intraretinal fluid-IRF, subretinal fluid-SRF, or between the retinal pigment epithelium (RPE) and Bruch's membrane-sub RPE fluid) [3]. The diseases most frequently diagnosed by using OCT include macular degeneration, diabetic retinopathy, and glaucoma. An extension of this technique is angiography OCT (OCT-angiography, Angio-OCT), which enables three-dimensional imaging of the retinal and choroidal vasculature without contrast [4]. Angio-OCT may be used in patients with age-related macular degeneration in order to qualify them for treatment [5]. The aim of the study is to compare classical methods of retinal vascular imaging such as fluorescein angiography (FA) with modern methods of ultrasound blood flow detection (Optical Coherence Tomographyangiography, Angio-OCT) in patients with AMD. 


\section{MATERIALS AND METHODS}

Images obtained during examination using AF, OCT, and Angio-OCT methods of four randomly selected patients (five eyes) treated for AMD in the Department of Ophthalmology Medical University of Silesia, Katowice, Poland were used for evaluation.

The study group embraced two women and two men aged from 49 to 90 years. Patients were treated with standard methods, and all performed tests were within the scope of routine check-ups in the treatment of AMD. Patients were informed about the exploitation of research results for scientific purposes and signed a consent required by law. The examination was performed using the following equipment: Topcon's 3D OCT (OCT), Topcon's Mark II TRC NW7SF (FA), and AngioVue from Optovue (Angio-OCT). All patients had best corrected visual acuity (BCVA) appraised.

\section{RESULTS}

Patient 1: a 79-year-old woman, right eye BCVA 0.3 . In fluorescein angiography there was increasing hyperfluorescence to the latter stages (Fig. 1). In optical coherence tomography fluid spaces were present in inner and outer layers of the retina (Fig. 2). In Angio-OCT the microvessels were visible in the choriocapillary layer (Fig. 3). Results obtained from this patient's OCT and especially Angio-OCT examinations yielded more detailed information concerning the changes in particular retinal layers and vessel structure. In comparison to fluorescein angiography, Angio-OCT scans were characterised by higher resolution.

Patient 2: a 58-year-old man, right BCVA 0.7. In fluorescein angiography we could observe increasing hyperfluorescence that covers pathological vessels, but in Angio-OCT the pathological microvessels were visible (Fig. 4). In comparison to fluorescein angiography, Angio-OCT examination enabled
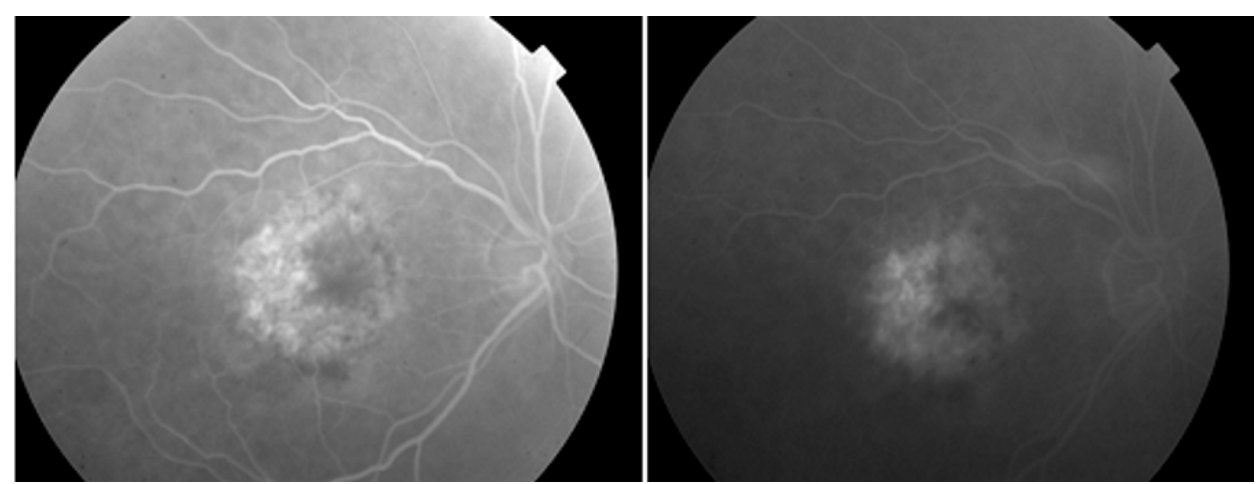

FIGURE 1. Patient 1. Fluorescein angiography image showing increasing hyperfluorescence in latter stages

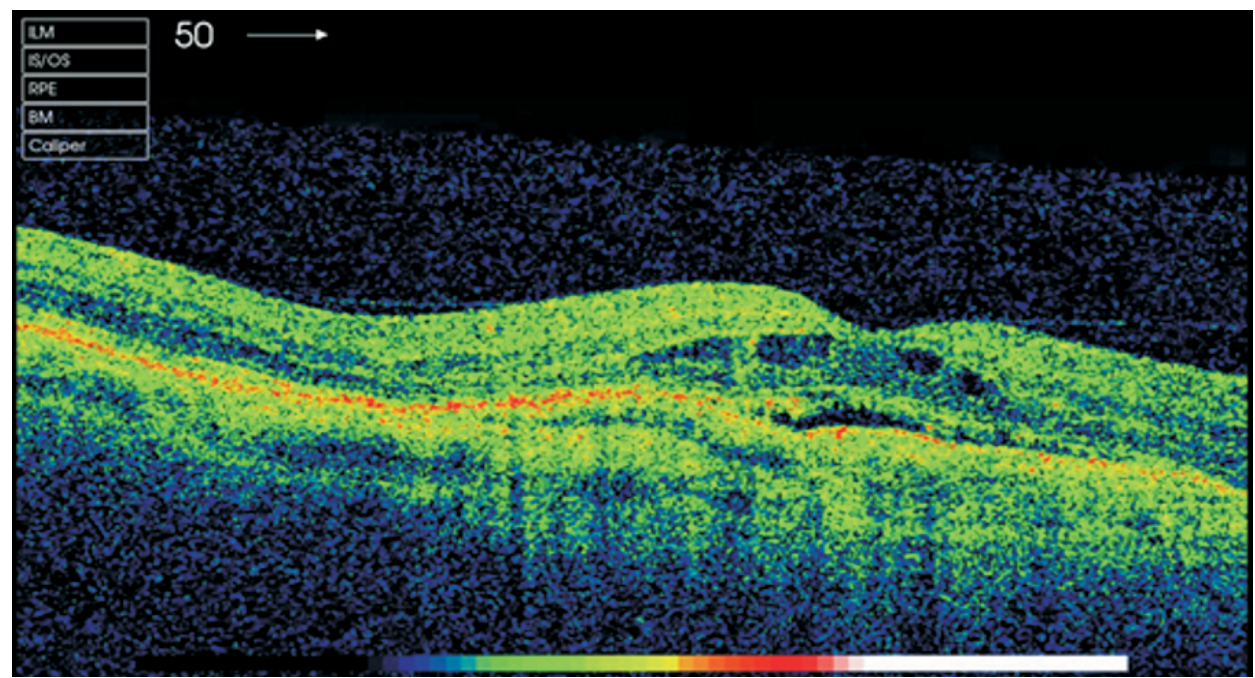

FIGURE 2. Patient 1. The OCT scan showing macular oedema and the fluid space is present (IRF and SRF) 


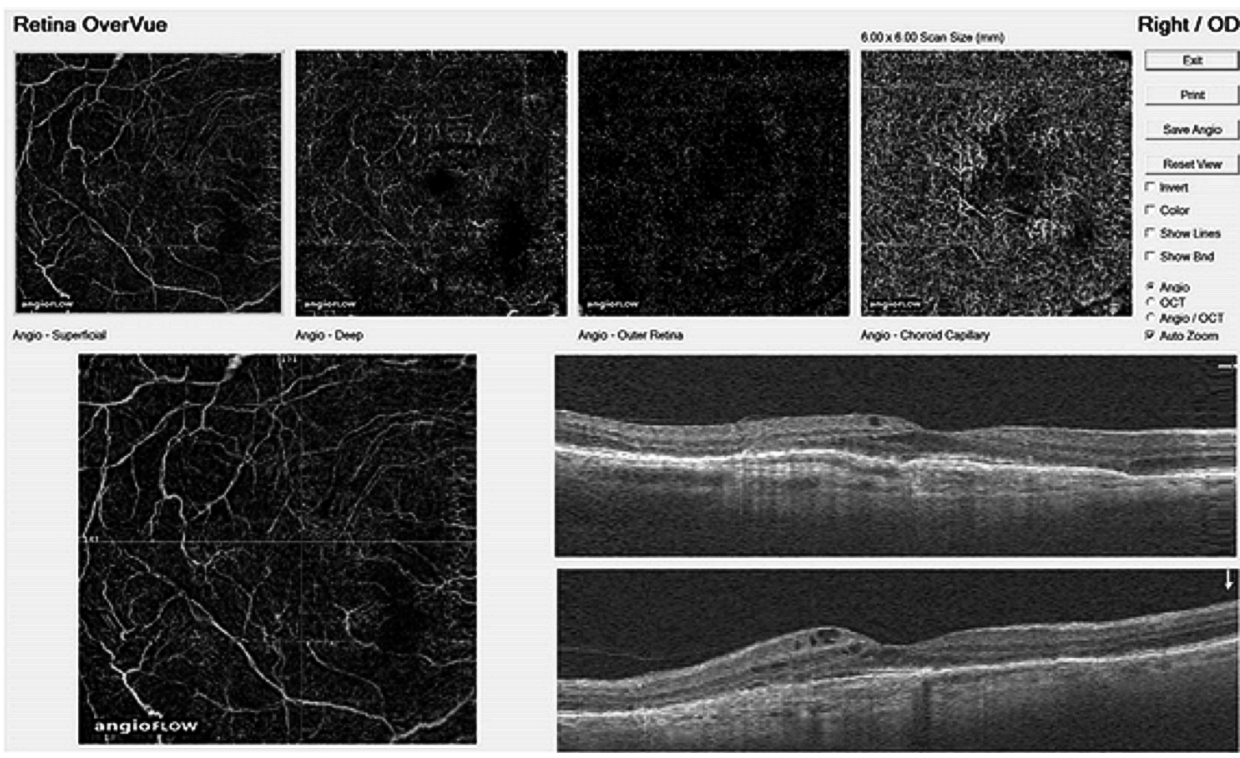

FIGURE 3. Patient 1. The Angio-OCT showing the same changes, and in the angio part there are micro vessels in the choriocapillary layer
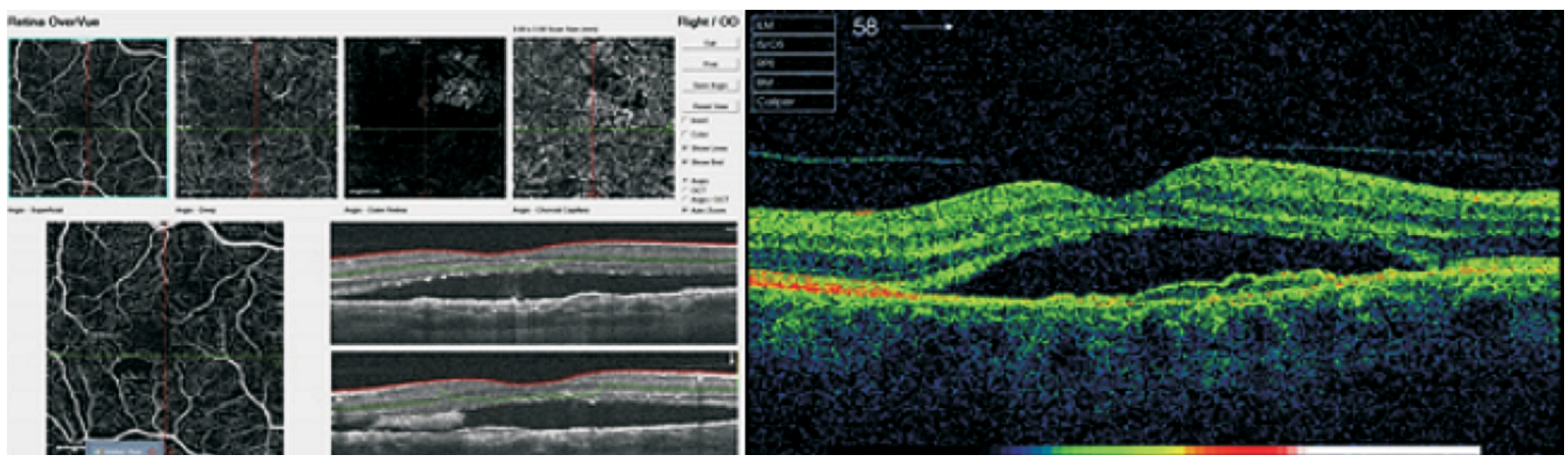

FIGURE 4. Patient 2. Angio-OCT scans showing that the structure of the inner layer of the retina is homogenous; below there is extensive fluid space in the outer retina. In the picture of the angio-part we can see pathological vessels in the deep layer of the retina and the choriocapillary layer

more precise evaluation of neovascular changes despite minor signs of the disease.

Patient 3: a 49-year-old man, left eye BCVA 1.0 and right eye BCVA 0.7. The patient reported himself to our clinic complaining about visual disorders in the right eye. Such disorders were not observed in the left eye (Figs. 5, 6). On OCT scans of the right eye we may observe loss of photoreceptors in macula. No changes were observed in the left eye OCT scans. We observed clear pigment epithelium detachment (PED) on Angio-OCT scans using wide field options. In the choriocapillary layer there was an excellent visualisation of pathological vessels (Fig. 7). OCT examination enabled diagnosis of the loss of photoreceptors in the macula. Only Angio-OCT examination enabled diagnosis and evaluation of the extent and severity of blood leakage as well as the location of pathological vessels. Angio-OCT examination carried out in vivo enables precise functional evaluation of retinal vascular changes.

Patient 4: a 90-year-old woman, right eye BCVA 0.1 , left eye BCVA 0.4. In fluorescein angiography there is increasing hyperfluorescence in the latter stages. In cases of fluorescein angiography, the hyperfluorescent area is visible covering the pathological vessels in the deeper layers of the retina whereas in Angio-OCT scans, thanks to the new possibilities of the device, excellent visualisation of microvessels in the choriocapillary layer was observed (Fig. 8). Fluorescein angiography appeared to be insufficient for examination of deeper retinal layers while Angio-OCT examination enabled evaluation of pathological vessels covered by fluorescent dye. 


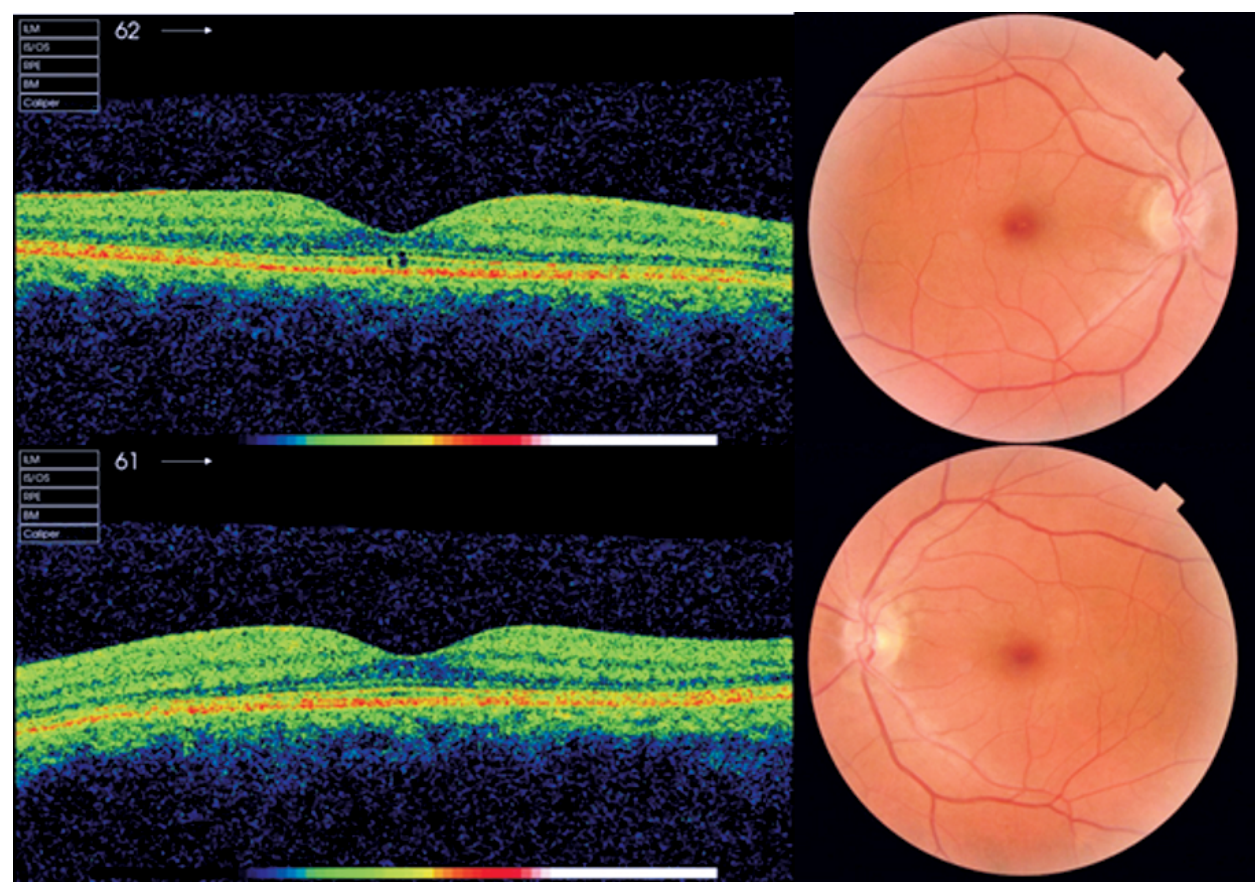

FIGURE 5. Patient 3. Comparison of OCT and FA. The patient has impaired vision in the right eye; there are no changes in eye fundus in the left eye; we can see the loss of the photoreceptors in macula in OCT in the right eye

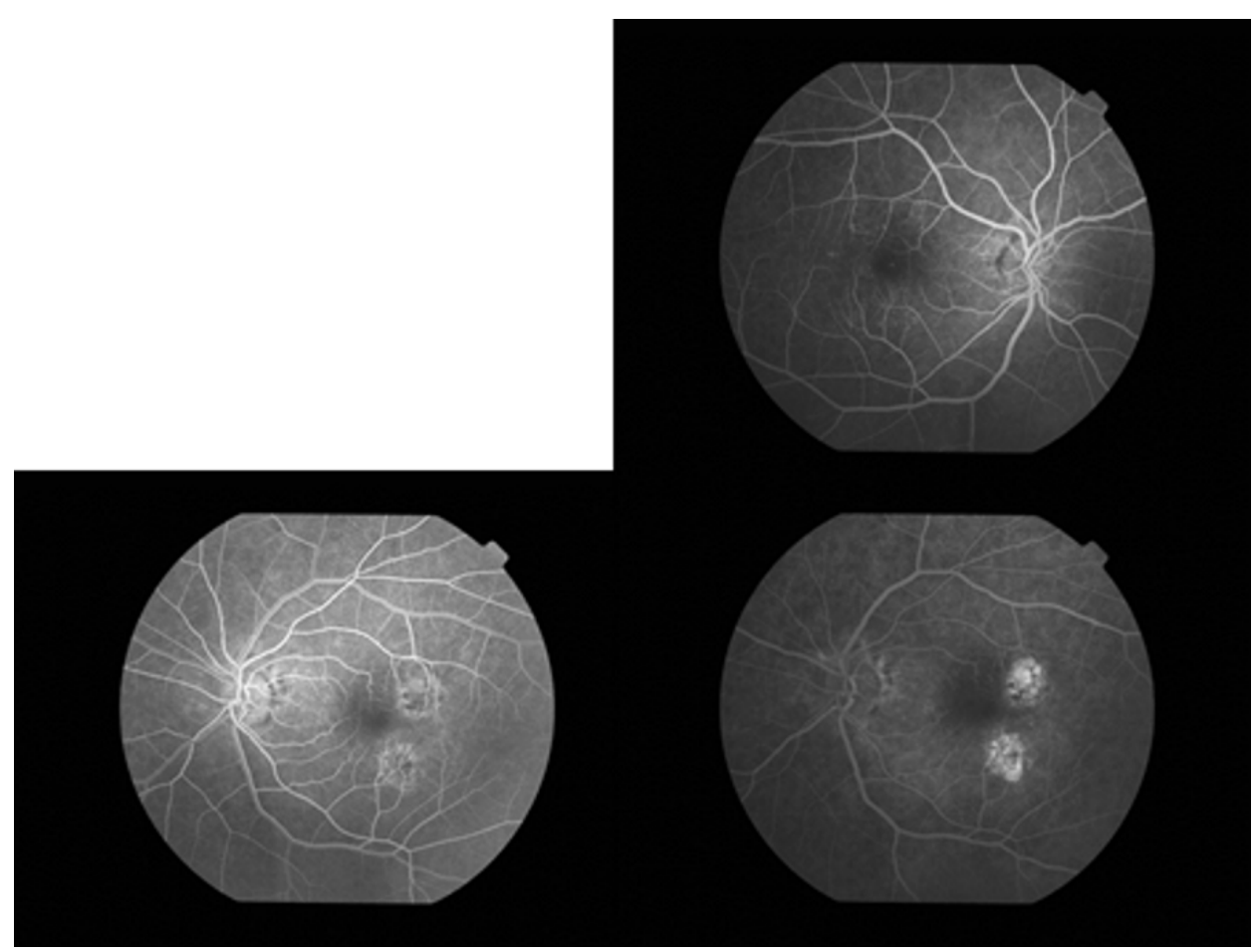

FIGURE 6. Patient 3. On fluorescein angiography we did not observe any pathological changes in contrast flow and we did not observe any leakage from vessels, but in the left eye we can observe two points of hyperfluorescence peripheral and temporal to the macula 


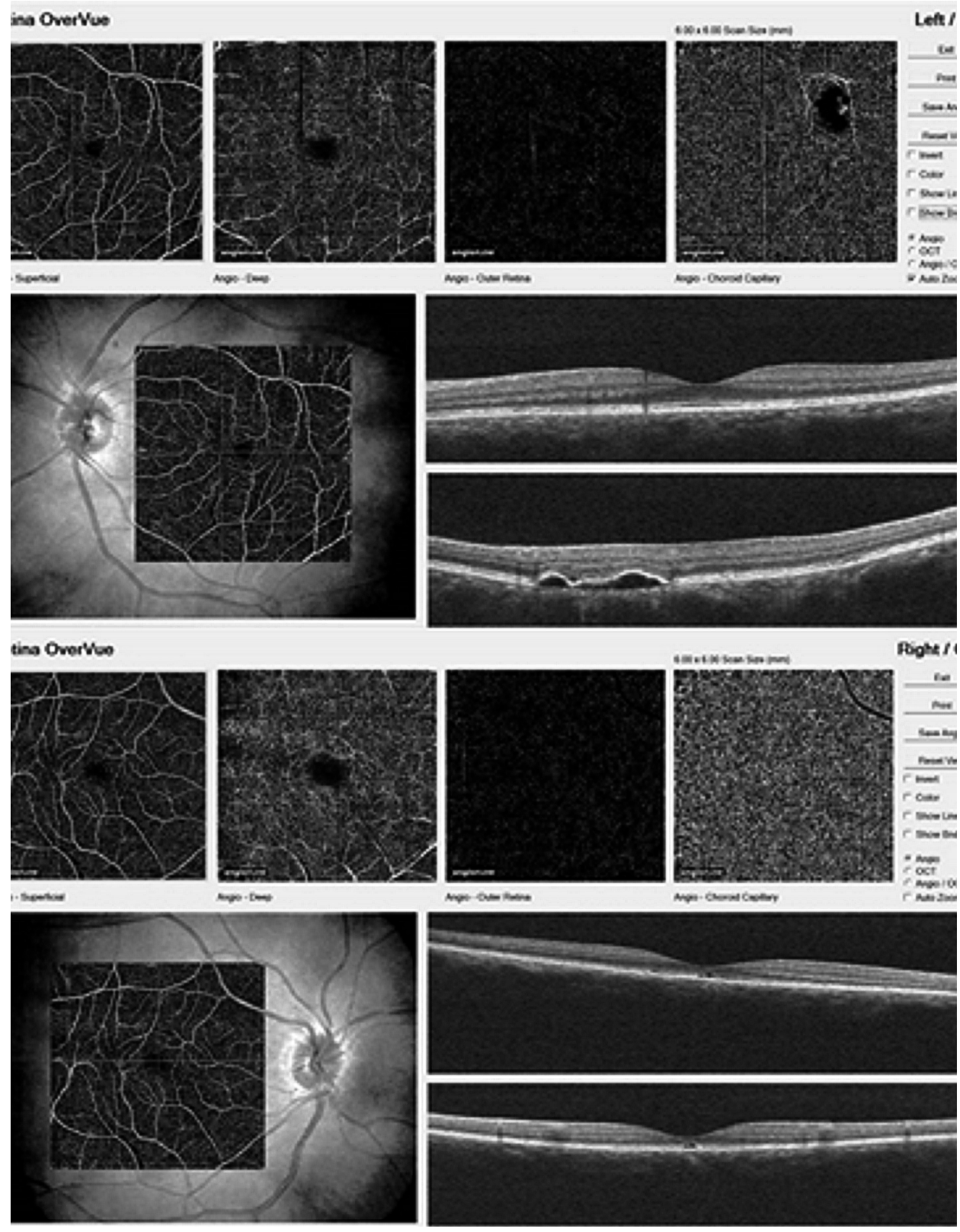

FIGURE 7. Patient 3. The Angio-OCT scans confirm the presence of pathological vessels in choroid in the left eye and we can see Pigment Epithelial Detachment (PED) on the OCT scans. Right eye losses in Retinal Pigment Epithelium (RPE)

\section{DISCUSSION}

A recognised classic method of diagnosing retinal vessels is AF. In this method, it is necessary to deliver a fluorescent dye to the human body. As a result of dye injection to the bloodstream the patient might be subject to side effects. Frequent complications associated with dye include: topical bleeding and petechiae, infections, and local and generalised allergic reactions. Rare complications associated with contrast include: renal dysfunction, myocardial infarction or brain damage, blood vessels damage through the cannula, or shock caused by an allergic or anaphylactic reaction. OCT and Angio-OCT are non-invasive and contactless test methods, so they can be used without exposing patients to complications $[6,7]$.

\section{CONCLUSIONS}

Angio-OCT is a non-invasive, non-contact, fast, and safe examination of patients treated for AMD. Angio-OCT is a modern method that provides important information about the progression of retinal diseases and helps to evaluate the vascular network in patients suffering from AMD. 


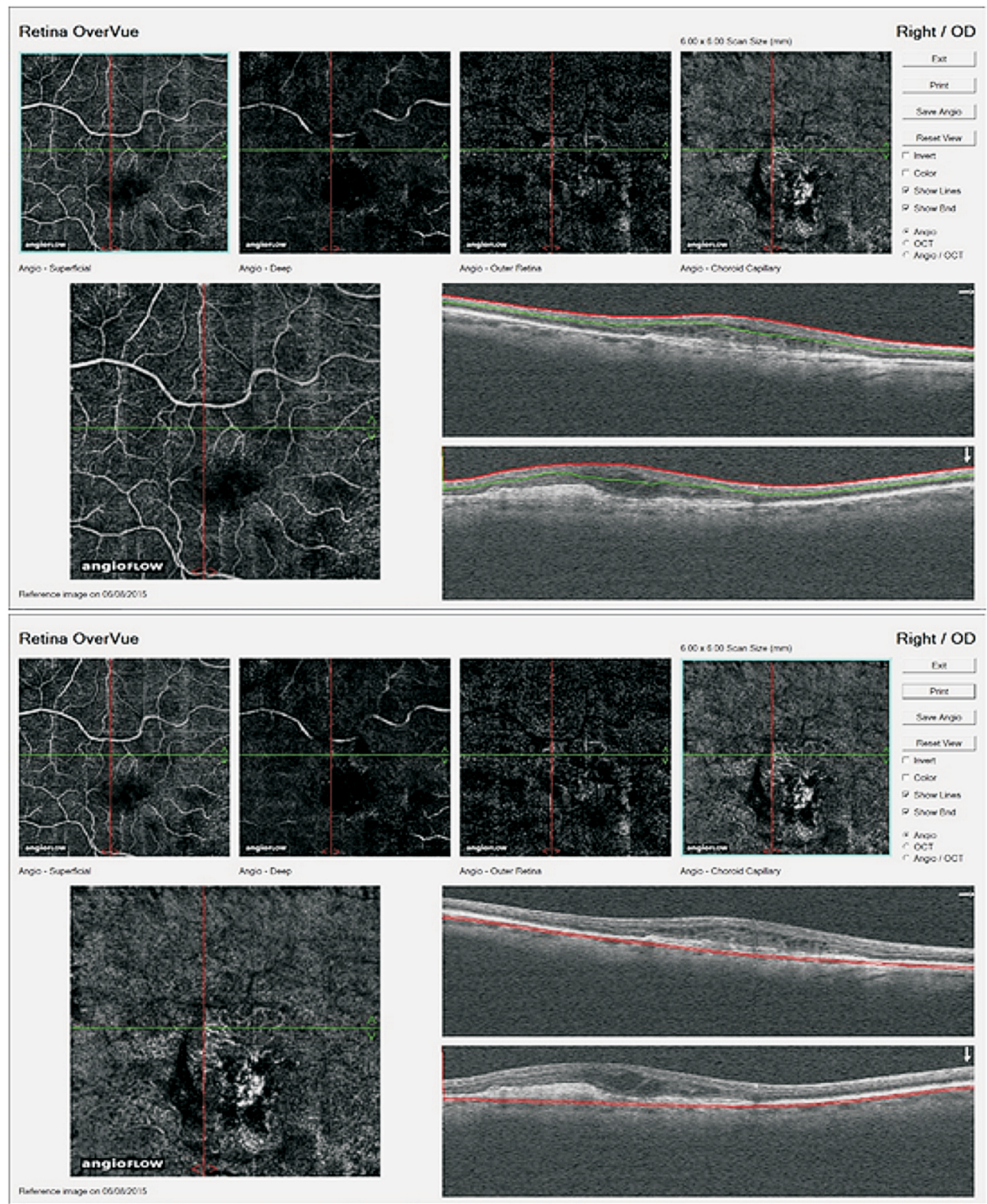

FIGURE 8. Patient 4. Angio-OCT scans showing microvessels in the choriocapillary layer

\section{CONFLICT OF INTEREST}

All authors declare that there is no conflict of interest. There are no sources of funding to declare.

\section{REFERENCES}

1. Bourne RR, Stevens GA, White RA et al. Vision Loss Expert Group: Causes of vision loss worldwide, 1990-2010: a systematic analysis. Lancet Glob Health 2013; 1: e339-e349. doi: 10.1016/S2214109X(13)70113-X.

2. Lim LS, Mitchell P, Seddon JM, Holz FG, Wong TY. Age-related macular degeneration. Lancet 2012; 379:1728-38. doi: 10.1016/ S0140-6736(12)60282-7.

3. Teussink MM, Breukink MB, van Grinsven MJ et al. OCT angiography compared to fluorescein and indocyanine green angiography in chronic central serous chorioretinopathy. Invest Ophthalmol Vis Sci 2015, 56 : 5229-5237. doi: 10.1167/iovs.15-17140.

4. Jia Y, Bailey ST, Hwang TS et al. Quantitative optical coherence tomography angiography of vascular abnormalities in the living human eye. Proc Natl Acad Sci USA 2015; 112: E2395-E2402. doi: 10.1073/ pnas. 1500185112.

5. Brown DM. Correlation Between Changes in OCT and Visual Acuity in the Management of Neovascular AMD. Retina Today 2013; 73-75.

6. Coscas G, Coscas F, Vismara S, Zourdani A, Li Calzi I. Optical Coherence Tomography in Age-Related Macular Degeneration. Springer, New York, USA- Heidelberg 2009.

7. Chen J, Lee L. Clinical applications and new developments of optical coherence tomography: an evidence-based review. Clinical and Experimental Optometry 2007; 90: 317-335. doi: 10.1111/j.14440938.2007.00151.x. 